\title{
On the measurement of employment intensity of agricultural growth
}

\begin{abstract}
Employment is the bedrock of a functioning economy for any nation wishing to progress and prosper. It is of great importance for policy makers to be able to measure the employment intensity of a nation's economy to identify regions of productive employment growth. Employment intensity has traditionally been measured in relation national economic growth typically as ratios. However, ratios are naturally biased and unbalanced. This does not allow the observer to take into account the differences in regions that may differ greatly in socioeconomic structure. For instance, some regions may be mired in poverty while others prosper greatly although both may show employment growth. This may instead indicate that employment is transferring from low productivity sectors into high productivity sectors instead of actual growth in the agricultural sector. Thus, we propose a new measure of employment growth focusing on the agricultural sector which we call the "Employment Intensity Index" which is symmetrical, proportional and scale invariant to regional differences. The index provides a single number that is comparable, simple and growth sensitive across all types of economic sectors across all differing policies within those sectors/regions regardless of the GDP/geographic size of sector/region. We then proceed to show its applicability.
\end{abstract}

Keyword: Employment intensity; Agriculture; Growth; Measurement 